Ketamin bringt Blase durcheinander

\section{Bei jugendlichem Pelvisschmerz an Drogen denken!}

- Bei der Jahrestagung der amerikanischen Urologen in San Francisco berichteten Mediziner aus Hongkong über ihre Erfahrungen mit 66 jugendlichen Teenagern und jungen Erwachsenen im Alter zwischen 13 und 25 Jahren. Fast alle nahmen das Anästhetikum Ketamin, einen NMDA-Rezeptor-Antagonisten, als Partydroge. Dass sich hinter den Problemen keine ernsthaften medizinischen Ursachen verbargen, schlossen die Urologen durch Nierenultraschall, Blasenscans nach dem Wasserlassen und Uroflowmetrie aus. Die positive Botschaft: Nach Ende der Drogenkarriere besserten sich die Symptom-Scores wieder. Die Blasenkapazität war nach einem Jahr weitgehend normalisiert. Fazit: Bei unklarer Ursache von urologischen Symptomen kann sich eine Anamnese der Partygewohnheiten von Jugendlichen lohnen.

wk

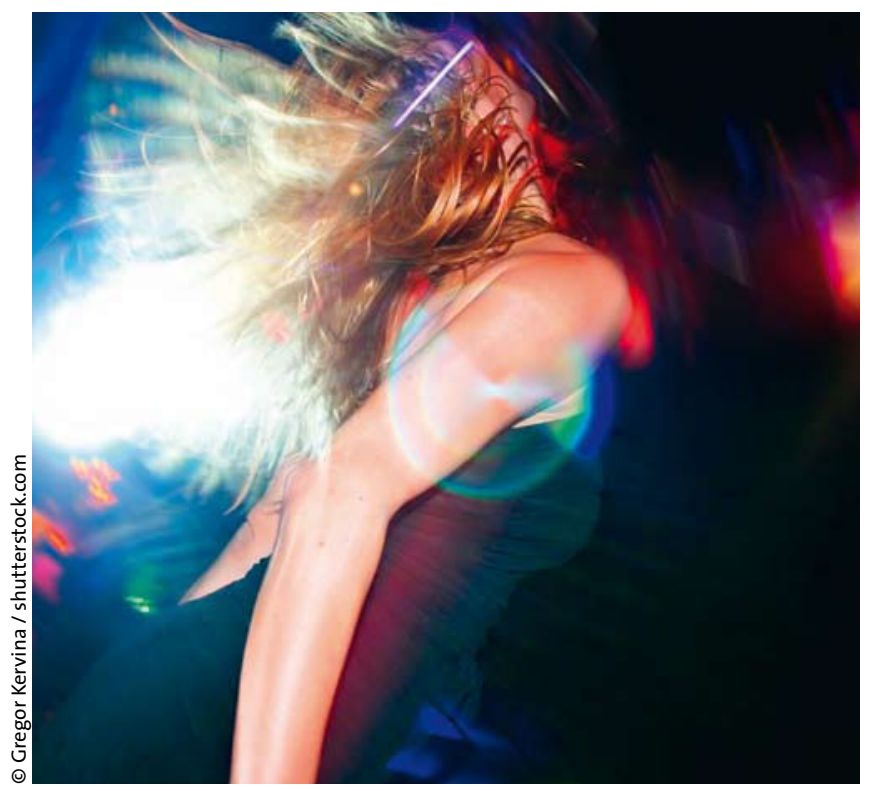

Bei Jugendlichen mit urologischen Symptomen unklarer Ursache sollte auch an den Konsum von Partydrogen gedacht werden.

\section{Aktuelle Daten}

\section{Alte Mediziner}

_ Die Zahl der im Beruf aktiven Ärzte, die schon über 60 Jahre alt sind, hat sich seit 1994 mehr als verdoppelt. Das geht aus einer aktuellen Statistik der Bundesärztekammer hervor-und unterstreicht wieder einmal die gravierenden Probleme, die auf die ambulante Versorgung zukommen. 1994 arbeiteten demnach 17.677 Ärzte über 60 Jahre; 2004 waren es bereits 36.233 , während die Zahl der jungen Ärzte in der Patientenversorgung um rund 1.000 zurückging. Bis 2015, so die Prognosen, werden rund 57.000 Ärzte in den Ruhestand treten. sv

\section{Chronisches Beckenschmerzsyndrom Stoßwellen lindern Schmerzen}

- Die extrakorporale Stoßwellentherapie (ESWT) bessert die Beschwerden beim chronischen Beckenschmerzsyndrom. In einer Studie wurden 60 Männer behandelt, die Hälfte erhielt einmal wöchentlich für vier Wochen hintereinander eine Niedrigenergie-ESWT mit 3.00o Impulsen, und zwar von einem perinealen Zugang ohne Anästhesie. Bei den übrigen Patienten erfolgte keine Energieübertragung. Danach registrierten die Forscher bei der Verum- im Vergleich zur Placebogruppe eine signifikante Verbesserung bei Schmerzen, Lebensqualität und Miktion [European Urology 2009; 56: 418]. ars
Sexsomnie und „Gähnologie“

\section{(Un-)freiwillige sexuelle Handlungen}

— Wer schläft, sündigt nicht? Von wegen. Eine kanadische Studie fand bei Patienten, die sich zur Abklärung von Schlafstörungen in ein Schlafzentrum begeben hatten, überraschend häufig eine bizarre Störung, die als „Sexsomnie“ bezeichnet wird. Die Betroffenen nehmen im Schlaf an sich selbst oder an ihrem Bettpartner sexuelle Handlungen vor. Diese können von der Masturbation bis zum gewaltsamen Geschlechtsverkehr reichen. In der Studie gaben nahezu $8 \%$ der Teilnehmer ( 63 von 832 ) in einem Fragebogen an, sich nachts unbewusst sexuell betätigt zu haben. Männer waren mit $11 \%$ fast dreimal so häufig „nachtaktiv“ wie Frauen (4\%). Die Betroffenen sprechen das Problem dem Arzt gegenüber nur selten an, bemerkte Koautorin Sharon A. Chung vom University Health Network in Toronto. In ihrer Untersuchung hatten nur vier der 832 Teilnehmer die peinliche Störung in der Sprechstunde beim Schlafspezialisten erwähnt.

Ungeklärt ist, ob die Bettpartner der Sexsomnie-Patienten, die unfreiwillig um den Schlaf gebracht werden, verstärkt gähnen. Denn wie Wolter Seuntjens im Rahmen der ersten internationalen Konferenz zum Gähnen in Paris postulierte, hat Gähnen nicht nur mit Müdigkeit oder Sauerstoffmangel zu tun, sondern auch mit Erotik und Sexualität. Schließlich seien Moleküle wie Oxytocin oder Stickstoffmonoxid sowohl beim Gähnen als auch bei sexueller Erregung involviert. Seuntjens Theorie zufolge gähnt man, wenn man etwas tun muss, was man nicht will oder wenn man etwas tun will, es aber nicht kann. Gähnt der Bettpartner, fällt es allerdings schwer zu unterscheiden, ob er unterbewusst zum Liebesspiel auffordert, ganz im Gegenteil gerade überhaupt keine Lust hat oder einfach nur müde ist. de/slx 